\title{
Microwave Small-signal modeling of FinFETs using Multi-parameter Rational Fitting Method
}

\author{
D. Deschrijver, G. Avolio, D. Schreurs, T. Dhaene, G. Crupi, and L. Knockaert
}

An effective approach based on a multi-parameter rational fitting technique is proposed to model the microwave small-signal response of active solid state devices. The model is identified by fitting multi-bias scattering-parameter measurements and its analytical expression is implemented in a commercial microwave circuit simulator. The approach has been applied to the modeling of a silicon based FinFET, and an excellent agreement is obtained between the measured data and model predictions.

Introduction: In order to continue the aggressive down-scaling of CMOS devices into the nanometer regime while counteracting short-channel effects, innovative strategies have been proposed in the last years. Amongst others, novel structures have been developed and as a result the FinFET has emerged as a promising solution $[1,2]$ that is compatible with the standard CMOS technological process. A lot of studies have been addressed to the extraction of reliable and accurate FinFET models in the microwave frequency range. Typically, compact models (CM) [3] or equivalent circuit models $(E C M)$ are employed to predict the microwave small-signal behavior of active devices and both approaches are based on multi-bias multifrequency scattering (S-) parameter measurements [4]. However, though being measurements-based, some knowledge about the physics of the device is still required. On the contrary, black-box or behavioral approaches can be completely independent on the device's physics and have a broad validity range. In order to calculate a model, the use of a multi-parameter rational fitting technique, called multivariate orthonormal vector fitting [5] is investigated. Although the method was originally designed for the macromodeling of passive linear systems, this letter shows 
that the approach can be promisingly applied to the compact modeling of active devices. Numerical results confirm that the calculated models are highly accurate. The models can be straightforwardly implemented in a circuit simulator.

Model description: Multi-parameter fitting techniques represent a successful strategy to approximate the behavior of structures and devices in the high-frequency range. Hence, the multi-bias scattering parameters $S_{i k}$ are approximated by a parameterized multivariate model. The model response changes as function of frequency $(f)$ and two electrical parameters: the gate voltage $\left(V_{G}\right)$ and drain voltage $\left(V_{D}\right)$.

$$
S_{i k}\left(f, V_{G}, V_{D}\right)=\frac{N\left(\theta, f, V_{G}, V_{D}\right)}{D\left(\theta, f, V_{G}, V_{D}\right)}=\frac{\sum_{n_{1}=1}^{P_{f}} \sum_{n_{2}=1}^{P_{V_{G}}} \sum_{n_{3}=1}^{P_{V_{D}}} c_{n_{1} n_{2} n_{3}} \Phi_{n_{1}}(f) \Psi_{n_{2}, n_{3}}\left(V_{G}, V_{D}\right)}{\sum_{n_{1}=1}^{P_{f}} \sum_{n_{2}=1}^{P_{V_{G}}} \sum_{n_{3}=1}^{P_{V_{D}}} \tilde{c}_{n_{1} n_{2} n_{3}} \Phi_{n_{1}}(f) \Psi_{n_{2}, n_{3}}\left(V_{G}, V_{D}\right)}
$$

The functions $\Phi$ and $\Psi$ are rational basis functions, which are defined as in [5]. They describe the frequency-dependent and parameter-dependent characteristics of the data, respectively. Note that the number of basis functions $(P)$ is chosen for each variable independently, according to the dynamic variation of the data. To identify the unknown model parameters $\theta^{t}=\left\{c_{n_{1} n_{2} n_{3}}^{t}, \tilde{c}_{n_{1} n_{2} n_{3}}^{t}\right\}$ in (1), the method in [5] is applied. It starts from an initial guess of model parameters $\theta^{0}$, and computes updated values in successive iteration steps $(t=0, \ldots, T)$ by solving several least-squares problems:

$$
\arg \min _{\theta^{t}}\left|\frac{N\left(\theta^{t}, f, V_{G}, V_{D}\right)}{D\left(\theta^{t-1}, f, V_{G}, V_{D}\right)}-\frac{D\left(\theta^{t}, f, V_{G}, V_{D}\right)}{D\left(\theta^{t-1}, f, V_{G}, V_{D}\right)} S_{i k}\left(f, V_{G}, V_{D}\right)\right|^{2}
$$

As the iteration proceeds, model (1) becomes an increasingly accurate approximation of the data. This process is repeated until convergence is reached. The procedure is well-known in literature as the Sanathanan-Koerner iteration, and an elaborate explanation can be found in [5] and [6]. By combining (2) with a suitable weighting scheme, the mean relative error per bias point can be minimized, 


$$
E_{i k}\left(V_{G}, V_{D}\right)=\frac{1}{N_{f}} \sum_{\text {freq }}\left|\frac{S_{i k}^{\text {meas }}\left(f, V_{G}, V_{D}\right)-S_{i k}^{\text {model }}\left(f, V_{G}, V_{D}\right)}{S_{i k}^{\text {meas }}\left(f, V_{G}, V_{D}\right)}\right|
$$

where $N_{f}$ corresponds to the number of the frequency samples per bias point.

Results: The investigated device is a triple gate FinFET fabricated in fully depleted silicon-on-insulator nMOSFET technology [4] with a $60 \mathrm{~nm}$ gate length and a $45.6 \mu \mathrm{m}$ gate width. Multi-bias and multi-frequency S-parameters have been measured with a vector network analyzer. The frequency was swept from $500 \mathrm{MHz}$ to $50 \mathrm{GHz}$ and both the $\mathrm{DC}$ gate $\left(\mathrm{V}_{\mathrm{G}}\right)$ and drain voltages $\left(\mathrm{V}_{\mathrm{D}}\right)$ were varied from $0 \mathrm{~V}$ to $1.2 \mathrm{~V}$. The extracted model has been implemented and simulated with the Advanced Design System $^{\odot}$ (ADS) circuit simulator. To verify the capability of the model to reproduce experimental results for the tested active solid-state device over a wide range of the input parameters, the comparison between measurements and simulations is illustrated in Figs. 1-3. Particularly, in Figs. 1-2 the modeled and measured Sparameters are shown as a function of the frequency and at two different bias conditions (i.e., $\mathrm{V}_{\mathrm{G}}=0 \mathrm{~V}$ and $\mathrm{V}_{\mathrm{D}}=0 \mathrm{~V}, \mathrm{~V}_{\mathrm{G}}=0.6 \mathrm{~V}$ and $\mathrm{V}_{\mathrm{D}}=0.6 \mathrm{~V}$ ).

In Fig. 3, the magnitude $S_{21}$ is reported as a function of the input parameters with $V_{G}$ fixed at $1.2 \mathrm{~V}$ in Fig. 3a, and $V_{D}$ fixed at $0.6 \mathrm{~V}$ in Fig. 3b. A good agreement between the experiments and the model simulations is achieved over a wide range of bias and frequency conditions, which further confirms the suitability of the multi-parameter rational fitting approach also for modelling active devices. In Table 1, the low values of the relative errors averaged over all the measured bias points and the maximum errors are reported to highlight the level of accuracy obtained with the proposed modeling approach.

Conclusion: A robust multi-parameter rational fitting technique has been exploited for modelling the microwave small-signal behaviour of FinFETs. A good agreement 
between model predictions and experimental data is achieved over a wide range of operating conditions. Furthermore, the model can be readily implemented in a commercial circuit simulator.

\section{References}

1 HISAMOTO, D., LEE, W.C., KEDZIERSKY, J., ANDERSON, E., TAKEUCHI, H., ASANO, K., TSU-JAE KING, BOKOR, J., and CHENMING, H.: 'A folded-channel MOSFET for deep-sub-tenth micron era', Electron Devices Meeting, 1998, pp. 1032 1034.

2 SUBRAMANIAN, V., PARVAIS, B., BORREMANS, J., MERCHA, A., LINTEN, D., WAMBACQ, P., LOO, J., DEHAN, M., GUSTIN, C., COLLAERT, N., KUBICEK, S., LANDER, R., HOOKER, J., CUBAYNES, F., DONNAY, S., JURCZAK, M., GROESENEKEN, G., SANSEN., W., DECOUTERE, S.: 'Planar Bulk MOSFETS Versus FinFETs: An Analog/RF Perspective', IEEE Trans. Electron Device, 2006, 53, (12), pp. 3071-3079.

3 SMIT, G.D.J., SCHOLTEN, A.J., SERRA, N., PIJPER, R.M.T., VAN LANGEVELDE, R., MERCHA, A., GILDENBLAT, G., and KLAASSEN, D.B.M.: 'PSPbased compact FinFET model describing dc and RF measurements', Electron Devices Meeting, 2006, pp. 1-4.

4 CRUPI, G., SCHREURS, D., PARVAIS, B., CADDEMI, A., MERCHA, A., and DECOUTERE, S.: 'Scalable and multibias high frequency modeling of multi-fin FETs', Solid-state Electronics, 2006, 50, (11), 1780-1786.

5 DESCHRIJVER, D., DHAENE, T., and DE ZUTTER, D.: 'Robust parametric macromodeling using multivariate orthonormal vector fitting', IEEE Trans. Microw. Theory and Tech., 2008, 56, (7), pp. 1661-1667.

6 SANATHANAN, C. K., KOERNER, J., 'Transfer Function Synthesis as a Ratio of Two Complex Polynomials', IEEE Transactions on Automatic Control, 1963, 48, (4), pp. 56-58. 


\section{Authors' affiliations:}

D. Deschrijver, T Dhaene and L. Knockaert (Department of Information Technology (INTEC), Ghent University, Gaston Crommenlaan 8, Gent, Belgium)

G. Avolio and D. Schreurs (Department of Electronic Engineering (ESAT) , K.U.Leuven, Kasteelpark Arenberg 10, Leuven, Belgium)

G. Crupi (Dipartimento di Fisica della Materia e Ingegneria Elettronica, University of Messina, Messina, 98166, Italy)

E-mail: dirk.deschrijver (@) intec.ugent.be

\section{Figure captions:}

Fig. 1 Frequency dependent S-parameters at $\mathrm{V}_{\mathrm{G}}=0 \mathrm{~V}$ and $\mathrm{V}_{\mathrm{D}}=0 \mathrm{~V}$ : model predictions (line) versus measurements (symbols)

Fig. 2 Frequency dependent S-parameters at $\mathrm{V}_{\mathrm{G}}=0.6 \mathrm{~V}$ and $\mathrm{V}_{\mathrm{D}}=0.6 \mathrm{~V}$ : model predictions (line) versus measurements (symbols)

Fig. 3 Magnitude of $S_{21}$ as a function of the input parameters. (a) $V_{G}=1.2 \mathrm{~V}$; (b) $V_{D}=$ $0.6 \mathrm{~V}$

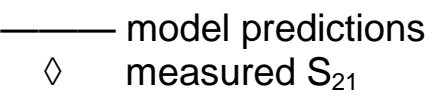

\section{Table captions:}

Table 1 Percentage average and maximum relative errors.

\section{Acknowledgements :}

This work was supported by the Fund for Scientific Research Flanders (FWO Vlaanderen). Dirk Deschrijver is a post-doctoral researcher of FWO Vlaanderen. 
Figure 1

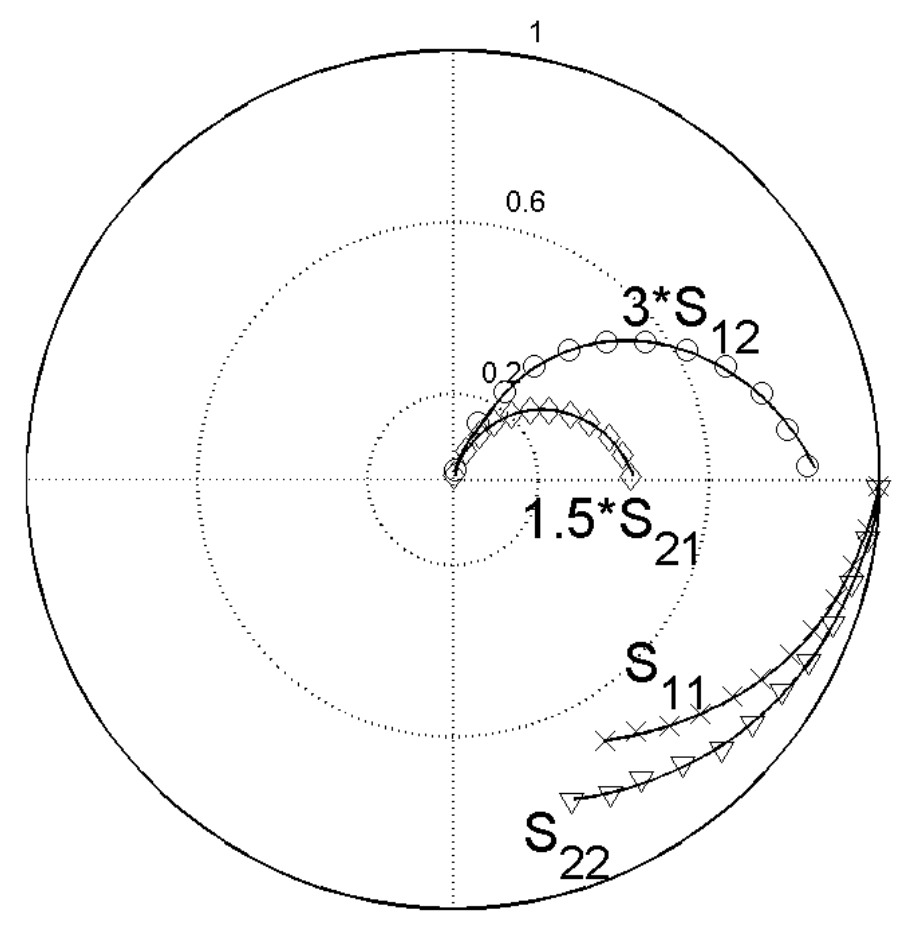


Figure 2

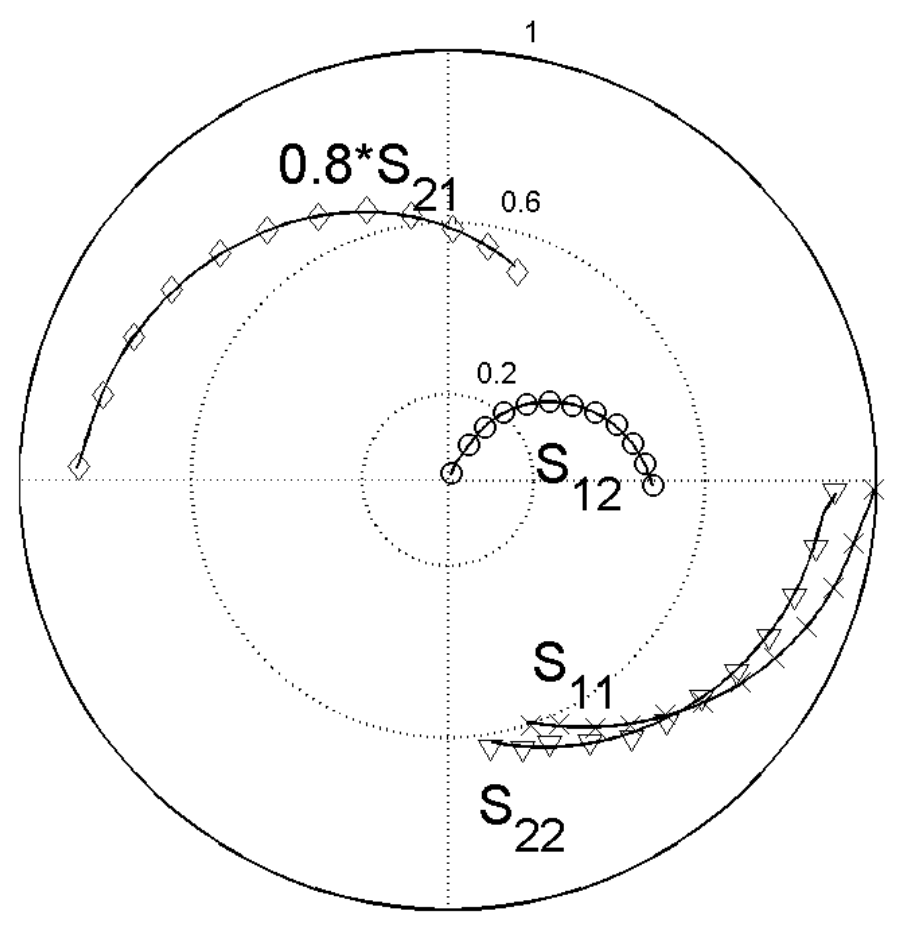


Figure 3

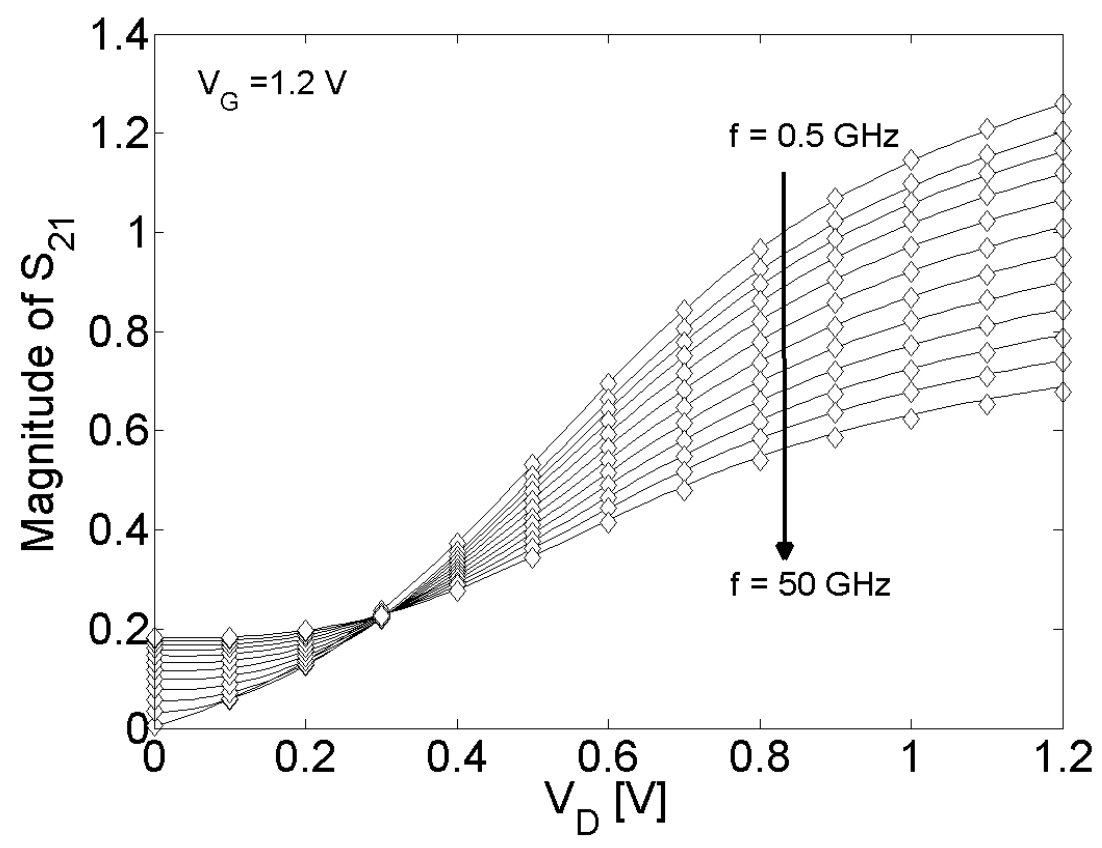

(a)

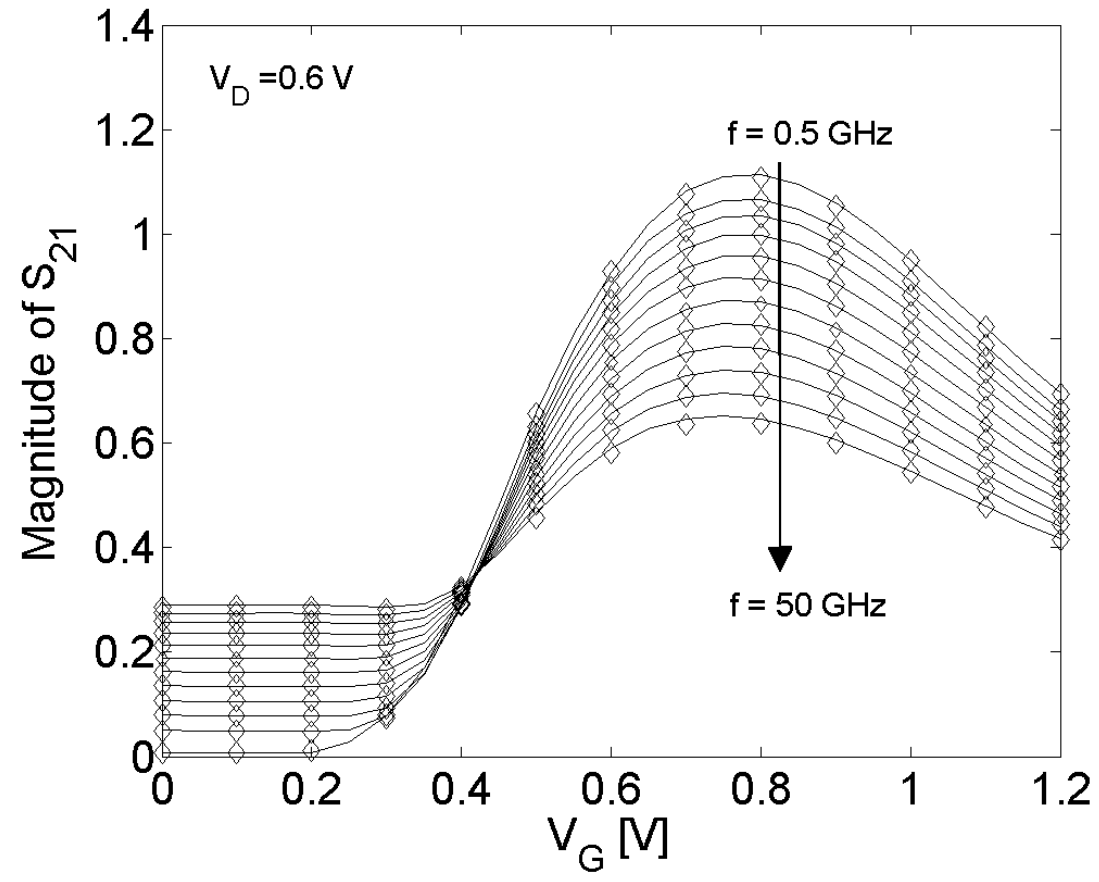

(b) 
Table 1

\begin{tabular}{|c|c|c|}
\hline & $\mathrm{E}_{\text {avg }}(\%)$ & $\mathrm{E}_{\max }(\%)$ \\
\hline $\mathrm{S}_{11}$ & 0.5 & 0.8 \\
\hline $\mathrm{S}_{12}$ & 1.0 & 2.5 \\
\hline $\mathrm{S}_{21}$ & 1.1 & 5.6 \\
\hline $\mathrm{S}_{22}$ & 0.7 & 3.3 \\
\hline
\end{tabular}

